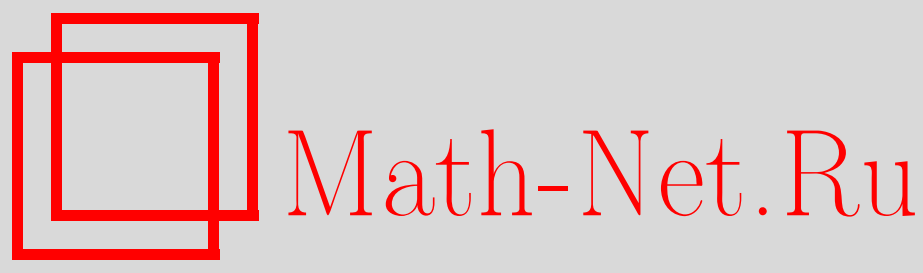

М. Б. Менский, Теоретико-групповой вывод релятивистского интеграла по путям и динамики "струн-историй", TMФ, 2012, том 173, номер 3, 392-415

DOI: https://doi.org/10.4213/tmf8372

Использование Общероссийского математического портала Math-Net.Ru подразумевает, что вы прочитали и согласны с пользовательским соглашением http://www.mathnet.ru/rus/agreement

Параметры загрузки:

IP: 54.209.52.79

26 апреля 2023 г., 18:28:01

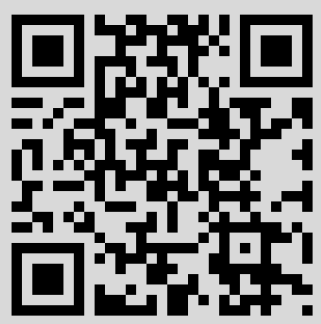




\title{
ФИЗИКА
}

Том 173, № 3

декабрь, 2012

(C) 2012 г.

\author{
М. Б. Менский*
}

\section{ТЕОРЕТИКО-ГРУППОВОЙ ВЫВОД РЕЛЯТИВИСТСКОГО ИНТЕГРАЛА ПО ПУТЯМ И ДИНАМИКИ "СТРУН-ИСТОРИЙ"}

\begin{abstract}
Фейнмановский интеграл по путям для релятивистских элементарных частиц выводится из теоретико-групповых соображений. Применяется подход, в рамках которого мы можем, задав группу (или полугруппу) симметрии, вывести из нее кинематику и динамику частицы, включая пространство состояний и пропагатор. Квантовые свойства частицы возникают в результате переплетения двух представлений (полу)группы симметрии, одно из которых описывает локальные свойства частицы, а второе - частицу как целое. Динамика в форме интеграла по путям возникает, если полугруппа симметрии имеет структуру, подобную релятивистскому аналогу группы Галилея (роль времени играет лоренц-инвариантное "собственное время"), но вместо трансляций включает полугруппу траекторий (параметризованных путей). Классическое действие, входящее в весовой функционал интеграла по путям, определяется заданием полугруппы с точностью до взаимодействия с калибровочным и/или гравитационным полем. Полученный формализм может представлять не только точечные частицы, но также нелокальные объекты типа "струн-историй”, которые, как показано ранее, позволяют объяснить удержание кварков.
\end{abstract}

Ключевые слова: интеграл по путям, симметрии, группа путей, путезависимые функции, квантовые струны.

\section{1. ВВЕДЕНИЕ}

Группы симметрии играют важную роль в теории элементарных частиц, в частности они используются для классификации частиц и для фиксации кинематики и динамики этих объектов. Вигнер [1] впервые предложил классифицировать элементарные частицы по неприводимым представлениям группы Пуанкаре. Затем Ньютон и Вигнер [2] в рамках теоретико-группового подхода проанализировали понятие локализации частицы. Окончательная формулировка этого понятия, использующая технику индуцированных представлений евклидовой группы, была дана Вайтманом [3].

* Физический институт им. П. Н. Лебедева РАН, Москва, Россия. E-mail: mensky@lpi.ru, mensky@mail.ru 
В работах [4], [5] был развит подход к теории элементарных частиц, который условно можно назвать теоретико-групповым выводом динамики. В этом подходе постулируется некоторая группа или полугруппа, имеющая структуру, аналогичную структуре группы Галилея, и выводится квантовая теория соответствующих элементарных объектов, например элементарных частиц (включая все элементы их кинематики и динамики). В рамках этого подхода пропагатор в пространстве-времени получается как оператор переплетения двух представлений группы, одно их которых (обычно неприводимое) описывает глобальные свойства частицы (представляет ее как целое), а второе - ее локализацию в пространстве-времени. Квантовый характер результирующей теории возникает именно из-за того, что локальные и глобальные свойства элементарного объекта определяются не одним и тем же, а двумя разными представлениями группы, а постоянная Планка возникает как параметр одного из этих представлений.

В работах [4], [5] этот подход применялся к описанию релятивистских частиц в пространстве-времени Минковского (основой теории в этом случае была группа Пуанкаре), нерелятивистских частиц в пространстве с абсолютным временем (группа Галилея) и релятивистских частиц в пространстве-времени де Ситтера (группа де Ситтера). В работе [6] теория нерелятивистских частиц во внешнем поле была выведена в рамках той же методики, но с заменой трансляций пространства-времени на траектории в нем. Принципиальную роль при этом играла группа путей, которая появилась в рамках калибровочной теории и гравитации и позволяла адекватно описывать внутренне присущую этим теориями нелокальность (которая проявляется, например, в эффектах типа эффекта Ааронова-Бома).

Калибровочная теория является локальной в результате того, что в ней налагается дополнительное требование инвариантности относительно группы калибровочных преобразований. Однако вполне возможно, что фундаментальным для этой теории является путезависимый формализм, который изначально вводит нелокальность, но зато позволяет избежать нефизического произвола, связанного с выбором калибровки. Нелокальная теория технически является более сложной, но при этом изначально содержит в себе возможность описания таких глобальных эффектов, как эффект Ааронова-Бома и его обобщения, в том числе неголономность, свойственную гравитации.

Как было показано в работах Мандельстама [7], [8], калибровочную теорию и гравитацию можно сформулировать в явно калибровочно-инвариантной форме (т. е. устранить нефизический произвол, состоящий в выборе калибровки), если для описания состояний частиц вместо обычных волновых функций, зависящих от точки пространства-времени, использовать функции, зависящие от путей в пространствевремени. Оказалось, что путезависимый формализм можно представить как теорию представлений так называемой группь путей.

Понятие группы путей было введено автором [9], [10] как обобщение "группоида параллельных переносов", введенного Шювегешем [11]. Оказалось, что структура группы естественно возникает вместо структуры группоида, а группоид параллельных переносов - это лишь одно из представлений группы путей. Группа путей является обобщением группы трансляций на случай, когда обычная трансляционная инвариантность не имеет места (например, из-за наличия внешних полей) [9], [10]. Это позволило интерпретировать путезависимый формализм Мандельстама в терминах индуцированных представлений группы путей [10], [12], [13]. 
В настоящее время многое указывает на то, что и в теории сильных взаимодействий, и в квантовой гравитации какая-то форма нелокальной теории является более фундаментальной, а обычная локальная теория возникает лишь в определенных специфических условиях. Группа путей указывает на один из способов введения нелокальности. При этом есть основания надеяться, что этот способ окажется эффективным, так как группа путей оказалась естественным математическим инструментом для формулировки некоторых фундаментальных понятий квантовой гравитации. Например, в работе [14] в терминах этой группы был сформулирован квантовый принцип эквивалентности, а в книге [10] с помощью группы путей сформулирован и доказан неабелев аналог теоремы Стокса.

В той же книге и в особенности в ее (расширенном) переводе на японский язык показано, что вытекающая из группы путей модель кварков как своего рода нелокальных объектов, “струн-историй" ("history-strings"), автоматически приводит к конфайнменту кварков (см. гл. 8 в [10]). С другой стороны, в рамках КХД конфайнмент тоже описывается существованием струн, связывающих кварки, но возникают эти струны как вторичные объекты (как специальные конфигурации поля глюонов). Это указывает на дуальность двух моделей кварков: модели, в которой первична нелокальность кварков, и модели, в которой первичны локальные кварки, а их нелокальные свойства и конфайнмент возникают вторично. Возможно, что фундаментальной является именно первая (изначально нелокальная) модель.

Все это указывает на актуальность исследования группы путей, различных ее модификаций и их представлений, особенно в приложении к квантовой теории. В работе [6] группа путей (а точнее, полугруппа параметризованных путей, или траекторий) в трехмерном пространстве была использована для теоретико-группового вывода динамики нерелятивистской частицы. В настоящей статье мы продолжим эту линию исследований, перейдя к полугруппе траекторий в пространстве-времени. Это позволяет распространить данный подход на теорию релятивистских частиц и "струн-историй".

Чтобы реализовать эту программу, мы сначала перейдем от обычной группы Галилея к ее релятивистскому обобщению, а затем заменим в ней пространственновременные трансляции на траектории (параметризованные пути) в пространстве Минковского. Заметим, что релятивистское обобщение группы Галилея было предложено в работе [15]. В честь авторов этой работы мы будем использовать для этого обобщения термин группа Агаши-Романа-Сантилли $($ АРC).

Группа АРС действует в пятимерном пространстве $A P C$, которое получается добавлением к пространству-времени Минковского лоренц-инвариантного параметра $\tau$, который называют инвариантным временем или собственным временем. Собственное время играет по отношению к пространству-времени ту же роль, которую в нерелятивистской теории и в группе Галилея играет обычное время $t$ по отношению к трехмерному пространству. Релятивистское свойство причинности формулируется в этом случае как распространение лишь в сторону увеличения $\tau$, а формализм в обычном пространстве-времени (в частности, обычный причинный пропагатор) получается затем интегрированием по всем положительным значениям параметpa $\tau$ [16].

Если применить теоретико-групповой вывод динамики непосредственно к группе АРС, то в результате получается обычная теория свободной релятивистской точеч- 
ной частицы, в том числе ее пропагатор ${ }^{1)}$. Для вывода релятивистского интеграла по путям и теории частицы во внешнем поле следует от группы AРC перейти к ее обобщению, заменив трансляции на траектории. При этом фактически получается теория нелокальных объектов (“струн-историй”), которые лишь в специальных условиях проявляются как точечные частицы.

Исходным пунктом для этого построения будет служить обобщенная полугруппа AРC, которая возникает, если сдвиги в пространстве-времени заменить на траектории в нем ${ }^{2)}$. Эта процедура получается непосредственным переносом соответствующей нерелятивистской схемы [6] с заменой пространства на пространство-время, а параметра времени $t$ нерелятивистской теории - на собственное время $\tau$. В результате вместо пространственно-временных трансляций будут фигурировать траектории в пространстве-времени, параметризованные параметром $\tau$. Это дает описание релятивистских частиц релятивистским интегралом по путям. Весовой функционал, определяющий меру интегрирования, автоматически оказывается равным экспоненте от классического действия, причем кинетическая часть лагранжиана определяется однозначно, а дополнительные члены, в силу требования инвариантности, обязательно должны иметь вид взаимодействия с калибровочным и/или гравитационным полем.

Заметим, что полученный результат можно интерпретировать не только как теоретико-групповой вывод динамики в форме интеграла по путям, но также и как вывод классического действия. Самое же главное, на наш взгляд, состоит в том, что построенный таким образом вывод интеграла по путям указывает на фундаментальный характер путей и на фундаментальную роль нелокальных объектов (локализуемых не в пространстве точек, а в пространстве путей) вместо локальных частиц.

Статья организована следующим образом. В разделе 2 формулируется процедура теоретико-группового вывода динамики. В разделе 3 описывается группа АРС и из нее с помощью сформулированной процедуры выводится динамика свободной релятивистской частицы, включая ее пропагатор. В разделе 4 заменой трансляций на траектории определяется обобщенная полугруппа АРС и из нее выводится теория (вообще говоря, нелокального) элементарного объекта, состояния которого описываются путезависимыми функциям; затем в предположении, что существенной является лишь зависимость от точек (концов траекторий), выводится динамика точечной релятивистской частицы во внешнем калибровочном и/или гравитационном поле. Наконец, в разделе 5 мы подводим итоги и отмечаем, что результаты можно использовать как теорию нелокальных квантовых объектов типа "струн-историй".

\section{2. ТЕОРЕТИКО-ГРУППОВОЙ ВЫВОД КВАНТОВОЙ ДИНАМИКИ}

В этом разделе мы сформулируем основные стадии процедуры, которая позволяет, постулируя некоторую группу (или полугруппу), вывести из нее кинематику и динамику соответствующего элементарного квантового объекта. В общем случае это нелокальный объект. Для простоты изложения мы часто будем называть

\footnotetext{
1) Зная пропагаторы различных частиц и используя технику графов Фейнмана, можно по теории возмущений найти эффекты от различных взаимодействий.

2) Полугруппа траекторий (параметризованных путей) отличается от группы путей тем, что по-разному параметризованные пути считаются различными.
} 
этот объект элементарной частицей. Это оправдано также и тем, что в некоторых специфических условиях нелокальные объекты проявляют себя как точечные частицы. Более того, вполне возможно, что те объекты, которые мы считаем точечными частицами (например, кварки), на самом деле в общем случае являются нелокальными объектами, и лишь в специальных условиях их нелокальные свойства становятся ненаблюдаемыми [10].

Основными инструментами, необходимыми для построения, кроме самой группы (или полугруппы) $G$, будут два ее представления $U_{\mathrm{loc}}(G)$ и $U_{\text {elem }}(G)$ и операторы $S_{1}$ и $S_{2}$, которые переплетают эти представления (коммутируют с ними), т. е. отображают пространство одного из представлений на пространство другого представления и обратно, не нарушая действия (полу)группы $G$. Каждое из двух представлений будет реализовано как индуцированное из соответствующей подгруппы (или подполугруппы) $H \subset G$ и $K \subset G$, что технически облегчает построение операторов переплетения.

Пространства, в которых действуют два характерных представления, в какой-то мере аналогичны координатному и импульсному представлениям, фигурирующим в квантовой механике, а операторы переплетения аналогичны прямому и обратному преобразованиям Фурье. Есть, однако, существенная разница. Координатное и импульсное представления квантовой механики представляют различными способами одно и то же пространство состояний квантовой системы. В нашем случае лишь пространство $\mathcal{H}_{\text {elem }}$, в котором действует представление $U_{\text {elem }}(G)$, интерпретируется как пространство реальных состояний. Векторы более широкого простран-

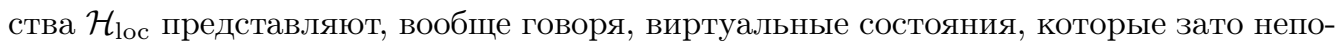
средственно характеризуют локализацию в пространстве-времени, и лишь оператор $S_{1}: \mathcal{H}_{\text {elem }} \rightarrow \mathcal{H}_{\text {loc }}$ проецирует пространство реальных состояний $\mathcal{H}_{\text {elem }}$ на подпространство в $\mathcal{H}_{\text {loc }}$, векторы которого описывают те же реальные состояния в форме, интерпретируемой в терминах локализации.

Чтобы проиллюстрировать роль виртуальных локализованных состояний, заметим, что пропагатор элементарной частицы понимается в КТП как двухточечная функция $K\left(x, x^{\prime}\right)$, которая описывает распространение этой частицы из одной точки пространства-времени в другую. Это можно трактовать как переход частицы из состояния, локализованного в точке $x^{\prime}$ пространства-времени, в состояние, локализованное в точке $x$. Каждое из состояний, локализованных в точке, является, разумеется, виртуальным ${ }^{3)}$. Такие виртуальные состояния описываются как векторы пространства $\mathcal{H}_{\text {loc }}$ В то же время при распространении частица движется как нечто целое, т. е. находится при этом в одном из своих реальных состояний (описываемых векторами из пространства $\left.\mathcal{H}_{\text {elem }}\right)$. Поэтому распространение должно

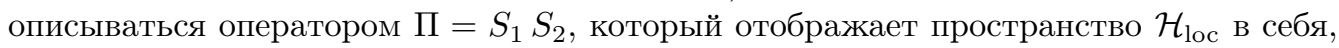
но вырезает при этом в нем подпространство, изоморфное $\mathcal{H}_{\text {elem }}$.

Ниже мы будем применять эту универсальную процедуру к различным группам (полугруппам). При этом мы будем стараться по возможности не вводить новых обозначений. В частности, группа (полугруппа), которая является исходным пунктом построения, каждый раз будет обозначаться одной и той же буквой $G$, а подгруппы (подполугруппы), из которых производится индуцирование, - буквами $H$

3) Именно поэтому при использовании пропагатора, скажем, в графе Фейнмана происходит интегрирование по всем значениям $x^{\prime}$ и $x$. 
и $K$. Однако следует помнить, что конкретное математическое определение (и отчасти физическая интерпретация) соответствующих объектов зависит от того, какая группа (полугруппа) $G$ выбирается в начале процедуры.

2.1. Вывод пропагаторов в теоретико-групповом подходе. В рамках теоретико-группового подхода к теории частиц [4], [5] пропагаторы могут быть получены из операторов переплетения специальных представлений группы (полугруппы) $U_{\mathrm{loc}}(G)$ и $U_{\mathrm{elem}}(G)$. Первое из них, $U_{\mathrm{loc}}$, описывает локальные свойства частицы. Оно должно быть индуцированным представлением из подгруппы (подполугруппы) $H \subset G$ такой, что факторпространство $H \backslash G$ описывает пространство-время или другое пространство локализации, так или иначе связанное с пространством-временем $^{4)}$. В этом случае векторы пространства $\mathcal{H}_{\text {lос }}$, в котором действует представление $U_{\text {loc }}(G)$, реализуются как функции на факторпространстве $H \backslash G$ и соответствуют состояниям, локализованным в той области, в которой сосредоточены эти функции [5]. Эти состояния в общем случае являются виртуальными.

Второе представление $U_{\text {elem }}$, характеризующее глобальные свойства частицы (ее элементарность), действует в пространстве реальных состояний. Например, в качестве $U_{\text {elem }}$ естественно выбрать неприводимое представление. Если это представление тоже реализовать как индуцированное из некоторой подгруппы (подполугруппы), то операторы переплетения обоих представлений легко строятся по общим правилам переплетения индуцированных представлений.

Пусть $S_{1} \in\left[U_{\text {elem }}, U_{\text {loc }}\right], S_{2} \in\left[U_{\text {loc }}, U_{\text {elem }}\right]$ - операторы, переплетающие эти представления, т. е. для любого элемента $g$ (полу)группы $G$ выполняются соотношения

$$
S_{1} U_{\text {elem }}(g)=U_{\text {loc }}(g) S_{1}, \quad S_{2} U_{\text {loc }}(g)=U_{\text {elem }}(g) S_{2} .
$$

Тогда оператор $\Pi=S_{1} S_{2}$ переплетает представление $U_{\text {loc }}$ с ним самим, проецируя его на подпредставление, эквивалентное $U_{\text {elem}}$. Интегральное ядро этого оператора является амплитудой перехода частицы из одной точки локализации в другую.

Причинный пропагатор получается из этой амплитуды с применением принципа причинности. В нерелятивистском случае этот принцип очень прост, он запрещает распространение из будущего в прошлое. Но в релятивистском случае все сложнее, и мы поговорим об этом в п. 2.6.

2.2. Индуцированные представления группы. Пусть дана группа $G$, ее подгруппа $K$ и представление этой подгруппы $\kappa(K)$, действующее в линейном пространстве $\mathcal{L}_{\kappa}$. Тогда индуиированное представление $U_{\kappa}(G)=\kappa(K) \uparrow G$ действует правыми сдвигами

$$
\left(U_{\kappa}(g) \varphi\right)\left(g^{\prime}\right)=\varphi\left(g^{\prime} g\right)
$$

в пространстве $\mathcal{H}_{\kappa}$ таких функций на $G$ со значениями в $\mathcal{L}_{\kappa}$, которые удовлетворяют так называемому структурному условию:

$$
\varphi(k g)=\kappa(k) \varphi(g) \quad \forall g \in G, \quad k \in K
$$

4)В частности, в разделе 4 это будет множество траекторий в пространстве-времени. 
Вместо функций на группе можно использовать функции на однородном пространстве $\mathcal{X}=K \backslash G$.

Обозначим через $x$ точку однородного пространства $\mathcal{X}$, т. е. соответствующий смежный класс. Для каждого смежного класса $x=K g \in \mathcal{X}$ выберем и зафиксируем некоторый элемент группы, который принадлежит этому классу. Обозначим его через $x_{G} \in x \subset G$. Этот элемент может служить представителем класса $x$, так что $x=K x_{G}$.

Выбор представителей классов в большой степени произволен, но если он тем или иным способом произведен, то это позволяет свести функцию на группе $\varphi$, удовлетворяющую структурному условию (2), к функции на множестве смежных классов.

Действительно, определим функцию на однородном пространстве формулой $\widetilde{\varphi}(x)=\varphi\left(x_{G}\right)$ (функция $\widetilde{\varphi}(x)$ зависит, таким образом, от конкретного выбора представителей). Тогда значение функции $\varphi$ на любом элементе группы $g$ с помощью структурного условия выражается через значение функции $\widetilde{\varphi}$ в соответствующем смежном классе: $\varphi(g)=\varphi\left(k x_{G}\right)=\kappa(k) \widetilde{\varphi}(x)$.

Инвариантное скалярное произведение в пространстве $\mathcal{H}_{\kappa}$ имеет форму

$$
\left(\varphi, \varphi^{\prime}\right)_{\kappa}=\int_{\mathcal{X}} d x\left\langle\widetilde{\varphi}(x), \widetilde{\varphi}^{\prime}(x)\right\rangle_{\kappa},
$$

где $\langle\cdot, \cdot\rangle_{\kappa}-$ инвариантное скалярное произведение в $\mathcal{L}_{\kappa}$, а $d x$ - инвариантная мера на пространстве $\mathcal{X}$.

Следовательно, вектор $\varphi \in \mathcal{H}_{\kappa}$ из пространства-носителя индуцированного представления может с равным правом представляться через функцию на группе $\varphi(g)$ или через функцию на однородном пространстве $\widetilde{\varphi}(x)$. Действие индуцированного представления может быть переписано в терминах функции $\widetilde{\varphi}(x)$.

Сделаем важное замечание относительно обозначений. В дальнейшем мы будем обозначать функцию на группе и соответствующую ей функцию на однородном пространстве одной и той же буквой $\varphi$. Какая из этих двух функций имеется в виду в любой последующей формуле, всегда будет видно из того, является ли аргумент функции элементом группы или точкой однородного пространства. Таким образом, $\varphi(g), g \in G,-$ это функция на группе, тогда как $\varphi(x), x \in \mathcal{X},-$ это функция на однородном пространстве. Если в тексте встречается буква $\varphi$ без указания аргумента, то речь идет о векторе пространства $\mathcal{H}_{\kappa}$, и не имеет значения, представляется ли этот вектор функцией на группе или функцией на однородном пространстве. Так, формулу для скалярного произведения в этих упрощенных обозначениях можно переписать в виде

$$
\left(\varphi, \varphi^{\prime}\right)_{\kappa}=\int_{\mathcal{X}} d x\left\langle\varphi(x), \varphi^{\prime}(x)\right\rangle_{\kappa}
$$

2.3. Переплетение индуцированных представлений. Пусть кроме $U_{\kappa}$ дано еще одно индуцированное представление $U_{\chi}(G)=\chi(H) \uparrow G$. Обозначим $\mathcal{Y}=H \backslash G$ и для каждого смежного класса $y \in \mathcal{Y}$ выберем его представитель $y_{G} \in y$. Вектор $\psi \in \mathcal{H}_{\chi}$ из пространства-носителя этого представления выражается или через функцию на группе $\psi(g)$, удовлетворяющую соответствующему структурному условию, или через функцию $\psi(y)$ на однородном пространстве $\mathcal{Y}$. 
Тогда оператор $S: \mathcal{H}_{\kappa} \rightarrow \mathcal{H}_{\chi}$, переплетающий два представления, $S \in\left[U_{\kappa}, U_{\chi}\right]$, т. е. удовлетворяющий условию $S U_{\kappa}(g)=U_{\chi}(g) S$, имеет вид [5]

$$
(S \varphi)(y)=\int_{\mathcal{X}} d x s\left(x_{G}\right) \varphi\left(x_{G} y_{G}\right),
$$

где семейство операторов $s(g): \mathcal{L}_{\kappa} \rightarrow \mathcal{L}_{\chi}$ удовлетворяет двустороннему структурному условию вида

$$
\chi(h) s(k g h) \kappa(k)=s(g) \quad \forall g \in G, \quad k \in K, \quad h \in H .
$$

2.4. Индуцированные представления полугруппы. Полугруппа отличается от группы тем, что не для всякого элемента имеется обратный элемент. В силу этого структура индуцированных представлений полугруппы имеет некоторые отличия от структуры индуцированных представлений группы.

Рассмотрим представление полугруппы $G$, индуцированное из подполугруппы $K \subset G$, и обозначим его как $U_{\kappa}(G)=\kappa(K) \uparrow G$. Это представление может быть определено теми же формулами $(1),(2)$, что и для индуцированного представления группы. Некоторые отличия имеются в определении факторпространства, инвариантной меры и переплетения индуцированных представлений.

Факторпространство $\mathcal{X}=K \backslash G$ следует определить как множество смежных классов $x=K g$. Полугруппа действует на нем правыми сдвигами, $g: K g^{\prime} \mapsto K g^{\prime} g$, но это действие интранзитивно. Пусть для каждого смежного класса $x \in \mathcal{X}$ выбран элемент (представитель) этого класса $x_{G}$ такой, что $x=K x_{G}$. Для любой функции $\varphi \in \mathcal{H}_{\kappa}$ положим $\varphi(x)=\varphi\left(x_{G}\right)$. С помощью структурного условия $(2)$ функция $\varphi(g)$ может быть выражена через $\varphi(x)$.

Для любого $k \in K$ имеем $K k \subset K$. Следовательно, $K k g$ есть подмножество в $K g$, но не обязательно совпадает с ним, потому что не всякий элемент полугруппы имеет обратный. Это значит, что в множестве смежных классов $\mathcal{X}$ существует частичная упорядоченность по включению.

Пусть $\overline{\mathcal{X}}$ - множество всех максимальных (относительно этого упорядочения) элементов в $\mathcal{X}$. В общем случае для любого $x \in \mathcal{X}$ существует максимальный класс, содержащий $x$, причем он не обязательно единственный. Мы, однако, ограничимся ситуацией, когда для каждого $x \in \mathcal{X}$ существует один и только один максимальный класс, включающий $x$. Обозначим его через $\bar{x}$. Сделаем это для всех элементов из $\mathcal{X}$. Тогда операция, обозначенная чертой, проецирует множество $\mathcal{X}$ всех классов на подмножество $\overline{\mathcal{X}}$ максимальных классов.

Из $x \subset \bar{x}$ следует, что $x_{G} \in K \bar{x}_{G}$. Следовательно, структурное условие (2) позволяет выразить $\varphi(x)$ через $\varphi(\bar{x})$. Таким образом, векторы пространства $\mathcal{H}_{\kappa}$ могут быть охарактеризованы функциями $\varphi(\bar{x})$ на множестве $\overline{\mathcal{X}}$.

2.5. Переплетение индуцированных представлений. Пусть $\mathcal{X}$ является $G$-пространством относительно полугруппы $G$, т. е. эта полугруппа действует на нем как полугруппа отображений, $g: x \mapsto x g$. Тогда инвариантная мера на $\mathcal{X}$ должна определяться формулой

$$
\int_{\mathcal{X}} d x F(x g)=\int_{\mathcal{X} g} d x F(x)
$$


$(\mathcal{X} g$ может отличаться от $\mathcal{X})$. В этом случае мы будем писать $d(x g)=d x$. В частности, $\mathcal{X}$ может совпадать с самой полугруппой $G$. Пусть инвариантная мера на $G$ факторизована как

$$
\int_{G} d g F(g)=\int_{\mathcal{X}} d x \int_{K} d k F\left(k x_{G}\right),
$$

где $d k$ - инвариантная мера на подполугруппе $K$. Тогда мера $d x$ на $\mathcal{X}=K \backslash G$ также инвариантна. Однако инвариантная мера на полугруппе может быть факторизована и иначе:

$$
\int_{G} d g F(g)=\int_{\overline{\mathcal{X}}} d \bar{x} \int_{K} d k F\left(k \bar{x}_{G}\right),
$$

где $d \bar{x}$ - мера на множестве $\overline{\mathcal{X}}$ максимальных классов. Тогда из инвариантности $d g$ и $d k$ следует, что $d \bar{x}$ инвариантна относительно операции $g: \bar{x} \mapsto \overline{\bar{x} g}$,

$$
\int_{\overline{\mathcal{X}}} d \bar{x} F(\overline{\bar{x} g})=\int_{\overline{\overline{\mathcal{X}} g}} d \bar{x} F(\bar{x}) .
$$

Мера $d \bar{x}$ может быть использована для построения переплетающего оператора $S \in$ $[\kappa(K) \uparrow G, \chi(H) \uparrow G]$. Можно показать, что оператор вида

$$
(S \varphi)(\bar{y})=\int_{\overline{\mathcal{X}}} d \bar{x} s\left(\bar{x}_{G}\right) \varphi\left(\bar{x}_{G} \bar{y}_{G}\right)
$$

является оператором переплетения, если операторы $s(g)$ удовлетворяют, как и в случае группы, двустороннему структурному условию (4).

\section{6. Пропагатор в собственном времени и причинный пропагатор.}

Сформулированная процедура позволяет из теоретико-групповых соображений вывести амплитуду распространения квантового объекта (его пропагатор). Однако также необходимо потребовать, чтобы распространение было причинным, т. е. чтобы получить причинный пропагатор. В случае нерелятивистской теории [6], когда имеется абсолютное время, требование причинности формулируется как распространение лишь вперед по времени. Но в релятивистском случае все несколько сложнее.

В случае отсутствия гравитационного поля, когда состояния можно разделить на положительно-частотные (относительно времени $x^{0}$ в пространстве Минковского) и отрицательно-частотные, принцип причинности можно сформулировать как распространение положительных частот в будущее, а отрицательных - в прошлое. Но в общем случае такое разделение невозможно. Вместо этого для формулировки причинного распространения в общем случае можно использовать формализм собственного времени, предложенный Штюкельбергом [16] и часто называемый формализмом Швингера-ДеВитта.

Согласно этому формализму кроме четырех пространственно-временных координат вводится вспомогательный пятый параметр $\tau$, который называется собственным временем или историческим временем. Теория строится по аналогии с нерелятивистской теорией, но с параметром $\tau$ вместо абсолютного (галилеевского) времени. Распространение релятивистского объекта параметризуется этим параметром, а причинный характер распространения означает, что движение происходит лишь 
в направлении его увеличения (в будущее относительно собственного времени). После нахождения пропагатора в собственном времени можно перейти к пропагатору в пространстве-времени, проинтегрировав (с соответствующим весом) по всем значениям параметра $\tau$.

Таким образом, если двухточечная функция $K_{\tau}\left(x^{\prime \prime}, x^{\prime}\right)$ (где аргументы являются точками в пространстве локализации) - это амплитуда распространения, зависящая от собственного времени $\tau$, то причинный пропагатор (уже не содержащий этого параметра) представляется интегралом по всем неотрицательным (в силу релятивистского принципа причинности) значениям $\tau$. Это дает для частицы массы $m$ следующее выражение ${ }^{5)}$ :

$$
K^{\mathrm{c}}\left(x^{\prime \prime}, x^{\prime}\right)=\int_{0}^{\infty} d \tau e^{-i\left(m^{2}-i \epsilon\right) \tau} K_{\tau}\left(x^{\prime \prime}, x^{\prime}\right) .
$$

Что касается амплитуды распространения $K_{\tau}\left(x^{\prime \prime}, x^{\prime}\right)$, зависящей от собственного времени, то она может быть записана в форме интеграла по путям. В разделе 4 выражение для этой амплитуды будет выведено из полугруппы, включающей траектории вместо пространственно-временных сдвигов.

\section{3. ВЫВОД РЕЛЯТИВИСТСКОЙ ТЕОРИИ ИЗ ГРУППЫ АРС}

Согласно работе [6] динамика нерелятивистской частицы в форме фейнмановского интеграла по путям может быть выведена, если постулировать полугруппу, которая получается при замене в группе Галилея сдвигов (трехмерного) пространства на траектории (параметризованные пути) в этом пространстве. Релятивистская динамика строится аналогично, но начать нужно с релятивистского обобщения группы Галилея, что и будет сделано в настоящем разделе.

Релятивистское обобщение группы Галилея было предложено в работах [15], [17], а ее неприводимые представления найдены и выражены в форме индуцированных представлений в работе [18]. Мы выведем здесь пропагатор свободной релятивистской частицы из этой группы, а в разделе 4 заменим в этой группе пространственновременные трансляции на траектории в пространстве-времени и в результате получим релятивистский интеграл по путям.

Группа АРС получается из группы Галилея, если трехмерное пространство заменяется на четырехмерное релятивистское пространство-время, а галилеевское абсолютное время - на пятый параметр релятивистской теории (собственное время) в смысле формализма Штюкельберга-Швингера-ДеВитта. Используя процедуру из раздела 2, мы найдем пропагатор, описывающий эволюцию с течением собственного времени. После этого переход к причинному пропагатору в четырехмерном пространстве-времени получается интегрированием по всем неотрицательным значениям собственного времени (см. п. 2.6).

3.1. Группа АРC. Рассмотрим структуру группы АРС, которая очень близка к структуре группы Галилея. Переход от группы Галилея к группе АРС, т. е. к релятивистскому аналогу группы Галилея, состоит в замене трехмерных век-

\footnotetext{
5) Мы используем естественные единицы измерения $\hbar=c=1$.
} 
торов $^{6)} \mathbf{x}, \mathbf{a}, \mathbf{v} \in \mathbb{R}^{3}$ на четырехмерные $x, a, v \in \mathbb{R}^{4}$ и вращений $r \in R$ на элементы группы Лоренца $\lambda \in \Lambda$. Подгруппа трансляций в группе APC содержит наряду с пространственно-временными трансляциями (на векторы $a \in \mathbb{R}^{4}$ ) также сдвиги пятого параметра, собственного времени $\tau$. Пятимерное пространство (пространство-время плюс собственное время) будем называть пространством АРС.

Группа АРС (которую мы в этом разделе будем обозначать буквой $G$ ) содержит подгруппу $T$ трансляций пятимерного пространства APC с элементами $(a, \tau)_{T} \in T$, подгруппу преобразований Лоренца $\lambda \in \Lambda$ и подгруппу собственных релятивистских преобразований Галилея $v_{G}$, характеризуемых векторами 4-скорости $v$. Произвольный элемент группы $G$ имеет вид $g=(a, \tau)_{T} \lambda v_{G}$, а умножение в группе полностью определяется, если заданы очевидные соотношения

$$
\begin{aligned}
(a, \tau)_{T}\left(a^{\prime}, \tau^{\prime}\right)_{T} & =\left(a+a^{\prime}, \tau+\tau^{\prime}\right)_{T}, \quad v_{G} v_{G}^{\prime}=\left(v+v^{\prime}\right)_{G}, \\
\lambda(a, \tau)_{T} \lambda^{-1} & =(\lambda a, \tau)_{T}, \quad \lambda v_{G} \lambda^{-1}=(\lambda v)_{G}
\end{aligned}
$$

и правила перестановки трансляций $(a, \tau)_{T}$ с релятивистскими аналогами собственных преобразований Галилея $v_{G}$ :

$$
v_{G}(a, \tau)_{T}=(a+\tau v, \tau)_{T} v_{G} .
$$

Все эти соотношения вполне аналогичны тем, которые имеют место для элементов группы Галилея.

Для дальнейшего (для вывода динамики) чрезвычайно существенно, что группа $G$ имеет проективные представления ("представления с точностью до числового множителя"), т. е. такие отображения $g \rightarrow U(g)$ элементов группы в множество операторов, для которых справедливо соотношение

$$
U(g) U\left(g^{\prime}\right)=f\left(g, g^{\prime}\right) U\left(g g^{\prime}\right),
$$

где $f\left(g, g^{\prime}\right) \in \mathbb{C}$ - некоторые комплексные числа, которые зависят от элементов группы $g, g^{\prime} \in G$ и называются мультипликаторами, или факторами данного представления. Из физических соображений не следует, что мультипликаторы должны быть равны единице, однако для некоторых классов групп преобразованием подобия операторов $U(g)$ можно обратить мультипликаторы в единицу и тем самым свести проективное представление к обычному, векторному, представлению. Но для группы Галилея и для группы АРС имеются такие проективные представления, которые не эквивалентны векторным. Можно лишь показать [18], что преобразованием подобия операторов представления можно добиться того, что мультипликаторы, или факторы $f\left(g, g^{\prime}\right)$ проективного представления в случае группы АРС всегда можно привести к виду

$$
f\left((a, \tau)_{T} \lambda v_{G},\left(a^{\prime}, \tau^{\prime}\right)_{T} \lambda^{\prime} v_{G}^{\prime}\right)=\exp \left[i m\left(\left(v, a^{\prime}\right)+\frac{1}{2}(v, v) \tau^{\prime}\right)\right],
$$

где $m$ - произвольный вещественный параметр и используется следующее обозначение для лоренцевой свертки 4-векторов:

$$
(v, a)=v^{0} a^{0}-\mathbf{v a} .
$$

6)Это может быть точка трехмерного пространства, вектор, на который производится сдвиг этого пространства, или скорость той системы отсчета, к которой производится переход при собственном преобразовании Галилея. 
Вместо явного рассмотрения проективных представлений группы АРС можно рассматривать обычные (векторные) представления другой группы, которая получается из группы АРС при помощи операции центрального расширения. Расширение производится путем включения в исходную группу вспомогательной однопараметрической группы $C$ с элементами $\xi_{C} \in C, \xi \in \mathbb{R}$, удовлетворяющими правилу умножения $\xi_{C} \xi_{C}^{\prime}=\left(\xi+\xi^{\prime}\right)_{C}$.

Произведем расширение группы АРС при помощи группы $C$, но будем для расширенной группы использовать то же обозначение $G$. Произвольный элемент расширенной группы АРС имеет вид $g=\xi_{C}(a, \tau)_{T} \lambda v_{G}$. Групповое умножение определяется условием, что любой элемент $\xi_{C} \in C$ коммутирует с любым элементом группы $\mathrm{APC}^{7)}$, соотношениями (8), а также следующими соотношениями, которые заменяют собой правила коммутации (9):

$$
v_{G}(a, \tau)_{T}=\xi_{C}(a+\tau v, \tau)_{T} v_{G}, \quad \xi=-\left((a, v)+\frac{1}{2} \tau(v, v)\right) .
$$

Множители типа $e^{i m \xi}$ в представлениях расширенной группы АРС играют роль мультипликаторов в самой группе АРС.

3.2. Представления (расширенной) группы АРС. Пятимерное пространство АРС (пространство-время плюс параметр собственного времени) является однородным пространством $H \backslash G$ группы $G$ по подгруппе $H$ с элементами $h=\xi_{C} \lambda v_{G}$. Представителями смежных классов могут служить чистые трансляции $(x, \tau)_{T}$. Соответствующий смежный класс имеет вид $H(x, \tau)_{T}$ и отвечает точке $(x, \tau)$ пространства-времени,

$$
(x, \tau)=H(x, \tau)_{T} \in H \backslash G .
$$

Локальное представление. Представление $U_{\text {loc }}$ (расширенной) группы APC должно быть индуцированным, $U_{\mathrm{loc}}=U_{\chi}=\chi(H) \uparrow G$, из подгруппы $H$, так как эта подгруппа является стабилизатором начала отсчета пространства APC (начало отсчета не меняется при действии элементов $h \in H)$. Выберем $\chi(h)=e^{i m \xi} \Delta(\lambda)$, где $\Delta-$ неприводимое представление группы Лоренца в пространстве $\mathcal{L}$. Представителями смежных классов по $H$ служат трансляции $(x, \tau)_{T}$, так что вектор пространства $\mathcal{H}_{\chi}$ определяется функцией $\psi(x, \tau)$ со значениями в $\mathcal{L}$.

Элементарное представление. Частица как целое описывается представлением $U_{\text {elem }}$, которое можно выразить как индуцированное, $U_{\text {elem }}=U_{\kappa}=\kappa(K) \uparrow G$. Подгруппу $K$ выберем как множество элементов вида $k=\xi_{C} \lambda(a, \tau)_{T}$, а представление $\kappa$ этой группы - в форме ${ }^{8)} \kappa(k)=e^{i m \xi} \Delta(\lambda)$.

Заметим, что в этом случае индуцированное представление, вообще говоря, не является неприводимым и может (в зависимости от конкретного выбора представления $\Delta$ ) описывать сразу несколько спинов. Выделение единственного спина производится в этом случае дополнительно, процедуру для этого мы обсудим в п. 3.4.

Представителями смежных классов группы $G$ по подгруппе $K$ могут служить элементы $v_{G}$, поэтому вектор пространства $\mathcal{H}_{\kappa}$ определяется функцией $\varphi(v)$ со значениями в $\mathcal{L}$.

\footnotetext{
7) А значит, он лежит в центре расширенной группы, отсюда название операции - центральное расширение.

8) Представления $\chi(H)$ и $\kappa(K)$ согласованы друг с другом так, чтобы существовало нетривиальное переплетение индуцированных представлений $U_{\text {loc }}$ и $U_{\text {elem }}$.
} 
3.3. Пропагатор свободной релятивистской частицы. Воспользовавшись тем, что локальное и элементарное представления группы $\mathrm{APC} U_{\chi}$ и $U_{\kappa}$ реализованы как индуцированные, и применяя общую формулу (3), можно представить операторы $S_{1} \in\left[U_{\kappa}, U_{\chi}\right], S_{2} \in\left[U_{\chi}, U_{\kappa}\right]$, переплетающие эти представления, в виде [5] ${ }^{9)}$

$$
\begin{aligned}
\left(S_{1} \varphi\right)(x, \tau) & =\int d^{4} v \exp \left[-i m\left((x, v)+\frac{1}{2} \tau(v, v)\right)\right] \varphi(v) \\
\left(S_{2} \psi\right)(v) & =\int d \tau \int d^{4} x \exp \left[i m\left((x, v)+\frac{1}{2} \tau(v, v)\right)\right] \psi(x, \tau) .
\end{aligned}
$$

Произведение этих операторов $\Pi=S_{1} S_{2}$ имеет вид

$$
\begin{aligned}
(\Pi \psi)(x, \tau) & =\int d \tau^{\prime} \int d^{4} x^{\prime} \Pi\left(x, \tau \mid x^{\prime}, \tau^{\prime}\right) \psi\left(x^{\prime}, \tau^{\prime}\right), \\
\Pi\left(x, \tau \mid x^{\prime}, \tau^{\prime}\right) & =\left(\frac{m}{2 \pi i\left(\tau-\tau^{\prime}\right)}\right)^{2} \exp \left(\frac{i m\left(x-x^{\prime}\right)^{2}}{2\left(\tau-\tau^{\prime}\right)}\right) .
\end{aligned}
$$

Ядро П $\left(x, \tau \mid x^{\prime}, \tau^{\prime}\right)$ этого оператора является амплитудой вероятности перехода частицы между двумя пространственно-временными точками [5]. Причина этого в следующем. Переход из одного виртуального состояния $\psi^{\prime} \in \mathcal{H}_{\chi}$, локализованного в пространстве-времени, в другое состояние $\psi \in \mathcal{H}_{\chi}$ происходит через одно из реальных состояний $\varphi \in \mathcal{H}_{\kappa}$, описывающих частицу как целое. Амплитуда перехода $\psi^{\prime} \rightarrow \varphi \rightarrow \psi$ равна произведению амплитуд переходов $\psi^{\prime} \rightarrow \varphi$ и $\varphi \rightarrow \psi$, которые в силу условия инвариантности равны соответственно $\left(\varphi, S_{2} \psi^{\prime}\right)_{\kappa}$ и $\left(\psi, S_{1} \varphi\right)_{\chi}$. Определяя полную амплитуду перехода $\psi^{\prime} \rightarrow \psi$ как сумму (по $i$ ) амплитуд переходов $\psi^{\prime} \rightarrow e_{i} \rightarrow \psi$, где $e_{i}-$ базисные векторы в $\mathcal{H}_{\kappa}$, получаем

$$
A\left(\psi, \psi^{\prime}\right)=\left(\psi, \Pi \psi^{\prime}\right)=\int d \tau \int d^{4} x \psi^{\dagger}(x, \tau)\left(\Pi \psi^{\prime}\right)(x, \tau) .
$$

Если $\psi, \psi^{\prime}$ - (обобщенные) состояния, локализованные каждое в единственной точке, то амплитуда перехода равна ядру оператора П.

Принцип причинности (распространение только из прошлого в будущее относительно параметра собственного времени $\tau$ ) дает причинный пропагатор свободной частицы, зависящий от собственного времени,

$$
\Pi^{\mathrm{c}}\left(x, \tau \mid x^{\prime}, \tau^{\prime}\right)=\theta\left(\tau-\tau^{\prime}\right)\left(\frac{m}{2 \pi i\left(\tau-\tau^{\prime}\right)}\right)^{2} \exp \left(\frac{i m\left(x-x^{\prime}\right)^{2}}{2\left(\tau-\tau^{\prime}\right)}\right),
$$

который совпадает с функцией Грина релятивистского уравнения Шредингера. Отсюда интегрированием по $\tau-\tau^{\prime}$ (по инвариантной мере) получается причинный пропагатор в пространстве-времени (см. п. 2.6):

$$
\Pi^{\mathrm{c}}\left(x, x^{\prime}\right)=\int_{0}^{\infty} d \tau\left(\frac{m}{2 \pi i \tau}\right)^{2} \exp \left(\frac{i m\left(x-x^{\prime}\right)^{2}}{2 \tau}\right) .
$$

9)Здесь использована естественная система единиц, в которой $\hbar=c=1$. При использовании обычных единиц вместо $m$ следует подставить выражение $m c / \hbar$. Постоянная Планка появляется в теории вместе с массой частицы при задании представления $\kappa(K)$. 
3.4. Выделение определенного спина. В основе построения, реализованного в настоящем разделе, лежал выбор неприводимого представления $\Delta(\Lambda)$ в качестве представления группы Лоренца $\Lambda$. Как известно, произвольная элементарная частица характеризуется массой $m$ и спином $j$, который может принимать значения $0,1 / 2,1,3 / 2,2, \ldots$. При этом в обычном формализме, как правило, фигурирует неприводимое представление группы Лоренца, которое в общем случае описывает более одного спина.

Произвольное неприводимое представление группы Лоренца характеризуется парой моментов $\left(l_{1}, l_{2}\right)$, каждый из которых может принимать значения $0,1 / 2,1$, $3 / 2,2, \ldots$. Представление группы Лоренца $\Delta_{\left(l_{1}, l_{2}\right)}$ описывает при этом спины $j=$ $l_{1}+l_{2}, l_{1}+l_{2}-1, \ldots,\left|l_{1}-l_{2}\right|$. "Лишние" спины исключаются в этом случае при помощи пары дифференциальных операторов $L_{j}, D_{j}$ (представляющих собой полиномы от $\left.\partial / \partial x^{\mu}\right)$, произведение которых пропорционально оператору Клейна-Гордона [5]. Пропагатор и волновая функция, описывающие спин $j$, и уравнения для волновой функции и пропагатора получаются с помощью таких операторов в виде

$$
\begin{aligned}
\Psi_{j} & =D_{j} \Psi, & \Pi_{j}^{\mathrm{c}} & =D_{j} \Pi^{\mathrm{c}}, \\
L_{j} \Psi_{j} & =0, & L_{j} \Pi_{j}^{\mathrm{c}}\left(x, x^{\prime}\right) & =\delta\left(x, x^{\prime}\right) .
\end{aligned}
$$

Здесь $\Psi$ - волновая функция, представляющая все спины $j=l_{1}+l_{2}, l_{1}+l_{2}-1, \ldots$ $\ldots,\left|l_{1}-l_{2}\right|$, входящие в неприводимое представление группы Лоренца, а функция $\Psi_{j}$ описывает лишь один из этих спинов, и аналогично для пропагатора.

Совершенно то же самое имеет место и в том формализме, который мы получили в рамках теоретико-группового вывода динамики. Это означает, что к пропагатору, найденному выше и описывающему, вообще говоря, несколько спинов, следует применить формулы (13) (предварительно построив нужные операторы $L_{j}$ и $D_{j}$ ).

\section{4. ВЫВОД ДИНАМИКИ В ПРОСТРАНСТВЕ ТРАЕКТОРИЙ}

В работах [10], [12], [13] была определена групnа путей (в пространстве Минковского), обобщающая пространственно-временные трансляции таким образом, чтобы инвариантность относительно этой группы не противоречила наличию внешних полей, с которыми взаимодействует частица. В указанных и более поздних работах на многих примерах было показано, что эта группа и ее представления составляют адекватный математический формализм для калибровочной теории и гравитации. Поэтому естественно попытаться обобщить процедуру, сформулированную в разделе 3, перейдя от трансляций к элементам группы путей.

Это будет сделано в настоящем разделе. Однако следует несколько уточнить сказанное. В определении группы путей элементы этой группы - пути - определяются как некоторые классы непрерывных кривых в пространстве-времени. Сейчас мы будем пользоваться более узкими классами кривых, которые можно назвать параметризованными путями или траекториями. Такие более узкие классы кривых образуют уже не группу, а полугруппу, полугруппу траекторий. Однако мультипликативная структура этой полугруппы согласуется со структурой группы путей (эти две алгебраические системы находятся в отношении гомоморфизма). Детали определений такого рода алгебраических систем можно найти в книге [10]. 
4.1. Полугруппа релятивистских траекторий. Пусть

$$
\{x\}_{\tau^{\prime}}^{\tau}=\left\{x(s) \in \mathcal{M} \mid \tau^{\prime} \leqslant s \leqslant \tau\right\}
$$

- непрерывная ${ }^{10)}$ кривая в пространстве Минковского $\mathcal{M}$. Определим произведение $\left\{x^{\prime \prime}\right\}_{\tau^{\prime \prime}}^{\tau}=\{x\}_{\tau^{\prime}}^{\tau} \cdot\left\{x^{\prime}\right\}_{\tau^{\prime \prime}}^{\tau^{\prime}}$ двух таких кривых, согласованных друг с другом в том смысле, что $x\left(\tau^{\prime}\right)=x^{\prime}\left(\tau^{\prime}\right)$ (конец одной из них совпадает с началом другой). Для этого положим

$$
x^{\prime \prime}(s)= \begin{cases}x^{\prime}(s), & \tau^{\prime \prime} \leqslant s \leqslant \tau^{\prime} \\ x(s), & \tau^{\prime} \leqslant s \leqslant \tau .\end{cases}
$$

Определенное таким образом умножение ассоциативно, так что множество всех таких кривых оказывается полугруппоидом (отличающимся от группы тем, что произведение определено не для всех элементов и обратный элемент не определен). Назовем его полугруппоидом параметризованных путей. Введем в этом полугруппоиде отношение эквивалентности, полагая $\{x\}_{\tau^{\prime}}^{\tau} \sim\left\{x^{\prime}\right\}_{\tau^{\prime}+\Delta \tau}^{\tau+\Delta \tau}$, если $x^{\prime}(s)=x(s-\Delta \tau)+a$ для всех значений $s$ и для заданных $a$ и $\Delta \tau$, и назовем возникающие в результате классы эквивалентности траекториями. Любые два класса содержат кривые, согласованные друг с другом в смысле совпадения конца одной кривой с началом другой. Поэтому произведение оказывается определенным для любых траекторий, и множество всех траекторий образует полугруппу траекторий.

Если $\{x\}_{\tau^{\prime \prime}}^{\tau^{\prime}}-$ кривая, то траектория, содержащая эту кривую, может быть охарактеризована скоростью $u(\sigma)=\dot{x}\left(\sigma-\tau^{\prime \prime}\right), 0 \leqslant \sigma \leqslant \tau=\tau^{\prime}-\tau^{\prime \prime}$. Обозначим эту траекторию через $[u]_{\tau}$. Таким образом, мы условимся характеризовать каждую траекторию кривой $[u]_{\tau}=\{u(\sigma) \in \mathcal{M} \mid 0 \leqslant \sigma \leqslant \tau\}$, точки которой имеют смысл скоростей. Произведение двух траекторий $\left[u^{\prime \prime}\right]_{\tau+\tau^{\prime}}=[u]_{\tau} \cdot\left[u^{\prime}\right]_{\tau^{\prime}}$ может быть выражено теперь формулой

$$
u^{\prime \prime}= \begin{cases}u^{\prime}(\sigma), & 0 \leqslant \sigma \leqslant \tau^{\prime}, \\ u\left(\sigma-\tau^{\prime}\right), & \tau^{\prime} \leqslant \sigma \leqslant \tau+\tau^{\prime}\end{cases}
$$

Введем также вырожденную траекторию $[u]_{0}$ со свойствами единицы.

Полугруппа траекторий обобщает группу трансляций. Точнее, существует гомоморфизм этой полугруппы в группу трансляций, переводящий траекторию $[u]_{\tau}$ в 4-вектор $a=\int_{0}^{\tau} d \sigma u(\sigma)$. Группа путей, определенная в работах [10], [12], [13], также может быть получена из полугруппы траекторий, если произвести еще одну факторизацию. При этой факторизации в один класс объединяются кривые, отличающиеся параметризацией (но проходящие через те же точки и в той же последовательности), а также добавлением "аппендиксов", т. е. отрезков, которые проходятся в одном направлении и вслед за этим - в обратном. Группа путей не понадобится нам в настоящей работе в явном виде.

10) Чтобы иметь возможность дифференцировать, рассматриваемые кривые должны быть также кусочно-дифференцируемыми. Мы, впрочем, не будем строго отслеживать в настоящей статье функционально-аналитические детали определений, сосредоточившись лишь на алгебраическом аспекте, т. е. способе умножения. 
Действие полугруппы траекторий на пространстве-времени естественно определить следующим образом. Если кривая $\{x\}_{\tau^{\prime \prime}}^{\tau^{\prime}}, x\left(\tau^{\prime}\right)=x^{\prime}, x\left(\tau^{\prime \prime}\right)=x^{\prime \prime}$, входит в класс $[u]_{\tau}, \tau=\tau^{\prime \prime}-\tau^{\prime}$, то положим

$$
\left(x^{\prime}, \tau^{\prime}\right)[u]_{\tau}=\left(x^{\prime \prime}, \tau^{\prime \prime}\right) .
$$

Такое действие траектории на точку пятимерного пространства АРС показывает, из какой точки нужно начать двигаться вдоль траектории $[u]_{\tau}$, чтобы попасть в данную точку $\left(x^{\prime}, \tau^{\prime}\right)$.

4.2. Обобщенная полугруппа АРС. Теперь мы обобщим группу АРС, заменив в ней трансляции на траектории $[u]_{\tau}$ из полугруппы траекторий, но так, чтобы получить алгебраическую систему, имеющую структуру типа группы Галилея, что необходимо для вывода динамики. Назовем эту систему обобщенной полугруппой $A P C$, но обозначать ее будем по-прежнему буквой $G$.

Преобразования Лоренца $\lambda \in \Lambda$ группы АРС останутся неизменными и будут играть в новой полугруппе такую же роль, какую они играли в группе АРС. Но преобразования перехода к движущейся системе отсчета придется существенно обобщить. Теперь соответствующий элемент будет описываться не одной 4-скоростью $v$, а целым семейством 4-скоростей, по одной для каждого момента собственного времени. Это семейство 4-скоростей и соответствующий элемент обобщенной полугруппы $\mathrm{APC} \mathrm{мы} \mathrm{обозначим} \mathrm{через}[v]=\left\{v(\sigma) \in \mathbb{R}^{4} \mid-\infty \leqslant \sigma \leqslant \infty\right\}$.

Произвольный элемент полугруппы $G$ можно представить в виде произведения $g=[u]_{\tau} \lambda[v]$. Чтобы определить произведение двух элементов такого вида, примем соотношения

$$
[v]\left[v^{\prime}\right]=\left[v+v^{\prime}\right], \quad \lambda[v] \lambda^{-1}=[\lambda v], \quad \lambda[u]_{\tau} \lambda^{-1}=[\lambda u]_{\tau},
$$

которые вместе с (15) составляют аналог соотношений (8). Вместо (9) положим

$$
[v][u]_{\tau}=[u+\tilde{v}]_{\tau}[\tilde{v}],
$$

где использовано обозначение $\tilde{v}(\sigma)=v(\sigma-\tau)$. Тогда элементы вида $g=[u]_{\tau} \lambda[v]$ образуют полугруппу, по структуре аналогичную группе АРС (и группе Галилея).

Полугруппа $G$ имеет нетривиальные проективные представления с мультипликаторами (факторами) вида

$$
f\left([u]_{\tau} \lambda[v],\left[u^{\prime}\right]_{\tau^{\prime}} \lambda^{\prime}\left[v^{\prime}\right]\right)=\exp \left[i m \int_{0}^{\tau^{\prime}} d \sigma\left(\left(u, v^{\prime}\right)+\frac{1}{2}\left(v^{\prime}, v^{\prime}\right)\right)\right] .
$$

Чтобы избежать введения проективных представлений, расширим нашу полугруппу при помощи группы $C$. Пусть элемент $\xi_{C} \in C$ этой группы коммутирует со всеми остальными элементами полугруппы $G$. Сохраним соотношения $(15),(17)$ и заменим правило перестановки (18) соотношением

$$
[v][u]_{\tau}=\xi_{C}[u+\tilde{v}]_{\tau}[\tilde{v}], \quad \xi=-\int_{0}^{\tau} d \sigma\left((u, \tilde{v})+\frac{1}{2}(\tilde{v}, \tilde{v})\right) .
$$

Назовем полугруппу с элементами $g=\xi_{C}[u]_{\tau} \lambda[v]$ (расширенной) обобщенной полугруппой $A P C$ и по-прежнему будем обозначать ее через $G$. Эта полугруппа будет 
основой для описания динамики релятивистской частицы во внешнем поле и даже динамики специфических нелокальных объектов (“струн-историй”). Для дальнейшего важно, что элементы вида $\xi_{C} \lambda[v]$ образуют подгруппу, т. е. для них определены обратные элементы.

4.3. Представления обобщенной полугруппы АРС. По аналогии с группой АРС определим элементарное представление обобщенной полугруппы АРС как индуцированное представление, $U_{\text {elem }}=U_{\kappa}=\kappa(K) \uparrow G$, из подполугруппы $K$ с элементами $k=\xi_{C} \lambda[u]_{\tau}$, а именно из представления этой подполугруппы $\kappa(k)=$ $e^{i m \xi} \Delta(\lambda)$, где $\Delta(\lambda)$ - неприводимое представление группы Лоренца. Смежные классы $x \in \mathcal{X}=K \backslash G$ имеют вид $x=K[u]_{\tau}[v]$, а максимальные классы $\bar{x}=K[v]$.

Заметим, что для каждого смежного класса существует единственный включающий его максимальный класс, так что корректно определена операция проецирования $x \rightarrow \bar{x}$ множества всех смежных классов $\mathcal{X}$ на множество максимальных смежных классов $\overline{\mathcal{X}}$. Следовательно, любая функция на полугруппе $\varphi(g)$ из пространства $\mathcal{H}_{\kappa}$ может быть сведена к функции на множестве максимальных смежных классов или к “функции от скорости” $\varphi[v]$. Закон преобразования этих функций, который следует из определения индуцированного представления, в явном виде нам не понадобится.

Определим локальное представление как $U_{\mathrm{loc}}=U_{\chi}=\chi(H) \uparrow G$, где $H$ - подгруппа, состоящая из элементов $h=\xi_{C} \lambda[v]$, а ее представление выбрано в виде $\chi(h)=e^{i m \xi} \Delta(\lambda)$. Факторпространство $\mathcal{Y}=H \backslash G$ состоит из классов $y=H[u]_{\tau}$. Множество максимальных классов $\overline{\mathcal{Y}}$ в данном случае совпадает с $\mathcal{Y}$ (так как все элементы $h \in H$ обратимы). Вектор из пространства $\mathcal{H}_{\kappa}$ представляется функцией от траекторий $\psi[u]_{\tau}$. Он описывает локализацию частицы в пространстве траекmорий.

Для локальных частиц пространство траекторий играет вспомогательную роль, позволяя вывести закон динамики в форме интеграла по путям. Ниже (в п. 4.7) мы увидим, как для таких частиц ввести волновую функцию в пространстве-времени. Тот факт, что мы начинаем с пространства траекторий, позволит выразить динамику частицы в форме интеграла по траекториям ${ }^{11)}$ и описать не только свободную частицу (как в случае группы AРC), но и частицу во внешнем поле. При этом окажется, что поле обязательно должно быть калибровочным и/или гравитационным.

Заметим, однако, что многое указывает на фундаментальный характер полугруппы траекторий, поэтому волновые функции в пространстве траекторий могут иметь самостоятельный физический смысл, описывая особого рода нелокальные объекты (“струны-истории”). По-видимому, некоторые важные наблюдаемые эффекты, в том числе конфайнмент цвета, можно объяснить нелокальным характером таких элементарных объектов. В то же время в определенных условиях нелокальные свойства этих объектов оказываются ненаблюдаемыми, и тогда эти объекты проявляют себя как точечные частицы ${ }^{12}$.

11) Обычно используется термин “интеграл по путям", но более точным является термин "интеграл по траекториям", если понимать траекторию так, как она определена выше.

12) Например, калибровочный дион описывается струной, положение которой существенно, если дион находится во внешнем калибровочном поле. Но если внешнее поле равно нулю, то существенно лишь положение самого диона (конца струны). В последнем случае калибровочный дион ведет себя как точечная частица [10]. 
4.4. Инвариантные меры. Представим инвариантную меру на $\mathcal{Y}=H \backslash G$ в форме

где

$$
\int_{\mathcal{Y}} d y F(y)=\int_{0}^{\infty} d \tau \rho(\tau) \int d[u]_{\tau} F\left(H[u]_{\tau}\right)
$$

$$
d[u]_{\tau}=\prod_{\sigma=0}^{\tau} \rho_{\tau}(\sigma, u(\sigma)) d^{4} u(\sigma) .
$$

Весовые функции $\rho$ и $\rho_{\tau}$ должны быть выбраны так, чтобы мера была инвариантна. Требование $d(y \lambda)=d y$ ее инвариантности относительно преобразований Лоренца дает $\rho_{\tau}=\rho_{\tau}\left(\sigma, u^{2}(\sigma)\right)$. Инвариантность относительно преобразований траекториями $d\left(y[u]_{\tau}\right)=d y$ дает $\rho=$ const и $\rho_{\tau}\left(\sigma, u^{2}(\sigma)\right)=\rho_{0}\left(u^{2}(\sigma)\right)$. Наконец, инвариантность относительно обобщенных собственных преобразований Галилея $d(y[v])=d y$ ведет к условию $d[u-v]_{\tau}=d[u]_{\tau}$, откуда имеем $\rho_{0}=$ const. Окончательно получаем

$$
\int_{\mathcal{Y}} d y F(y)=\int_{0}^{\infty} d \tau \int d[u]_{\tau} F\left(H[u]_{\tau}\right), \quad d[u]_{\tau}=\prod_{\sigma=0}^{\tau} d^{4} u(\sigma) .
$$

Последнюю формулу следует понимать как предел конечномерной аппроксимации с соответствующим нормировочным множителем. Разумеется, мы не обсуждаем здесь вопрос о сходимости, рассматривая все интегралы формально. Интеграл в (20) можно рассматривать, например, как предел гауссовой функциональной меры:

$$
\int d[u]_{\tau} F[u]_{\tau}=\lim _{\epsilon \rightarrow 0} \int \prod_{\sigma=0}^{\tau}\left[\left(\frac{\pi}{\epsilon}\right)^{2} d^{4} u(\sigma)\right] \exp \left[-\epsilon \int_{0}^{\tau} d \sigma u^{2}(\sigma)\right] F[u]_{\tau}
$$

Рассмотрим теперь факторпространство $\mathcal{X}=K \backslash G$ и соответствующее множество максимальных классов $\overline{\mathcal{X}}$. Как уже говорилось, последнее состоит из классов $K\left[v^{\prime}\right]$. Действие элементов вида $g=\xi_{C} \lambda[v]$ на максимальные классы переводит их опять в максимальные, не выводя из $\overline{\mathcal{X}}$. Однако действие элементов более общего вида переводит максимальный класс в немаксимальный (например, максимальный класс $K\left[v^{\prime}\right]$ становится немаксимальным при действии элемента $g=[u]_{\tau}$ ). Однако для каждого (вообще говоря, немаксимального) класса $x$ существует лишь один содержащий его максимальный класс $\bar{x}$. Тем самым на множестве всех классов определяется операция проецирования множества всех классов на множество максимальных классов. Если действие какого-то элемента группы превращает максимальный класс в немаксимальный, то операция проецирования снова переводит его в максимальный, например

$$
\overline{K[v][u]_{\tau}}=K[\tilde{v}], \quad \tilde{v}(\sigma)=v(\sigma-\tau) .
$$

Тем самым определяется операция $g: \bar{x} \mapsto \overline{\bar{x}}$. Нетрудно продемонстрировать (способом, аналогичным примененному выше), что мера на $\overline{\mathcal{X}}$, инвариантная относительно этой операции, имеет вид

$$
\int_{\overline{\mathcal{X}}} d \bar{x} F(\bar{x})=\int d[v] F(K[v]), \quad d[v]=\prod_{\sigma=-\infty}^{\infty} d^{4} v(\sigma) .
$$

Разумеется, все замечания, сделанные по поводу “меры" $d[u]_{\tau}$, остаются справедливы и в отношении $d[v]$. 
4.5. Переплетение представлений обобщенной полугруппы АРС. В соответствии с общей формулой (6) оператор $S_{1} \in\left[U_{\kappa}, U_{\chi}\right]$, переплетающий элементарное и локальное представления обобщенной полугруппы АРС, имеет вид

$$
\left(S_{1} \varphi\right)[u]_{\tau}=\int d[v] S_{1}[v] \varphi\left([v][u]_{\tau}\right)
$$

где операторы $S_{1}(g): \mathcal{L} \rightarrow \mathcal{L}$ удовлетворяют структурному условию (4). Как следствие этого условия имеем $S_{1}(v)=A=$ const, и этот оператор $A$ удовлетворяет соотношению $A \Delta(\lambda)=\Delta(\lambda) A$ для любого $\lambda \in \Lambda$, т. е. $A \in[\Delta, \Delta]$. В силу неприводимости представления $\Delta$ любой оператор, переплетающий это представление с ним самим, кратен единичному. Поэтому, опуская числовой множитель, примем $S_{1}(v)=1$. Применяя правило перестановки (19) к аргументу функции $\varphi$ в подынтегральном выражении и используя структурное условие $(2)$, можно привести $S_{1}$ к виду

$$
\left(S_{1} \varphi\right)[u]_{\tau}=\int d v \exp \left[-i m \int_{0}^{\tau} d \sigma\left((u, \tilde{v})+\frac{1}{2}(\tilde{v}, \tilde{v})\right)\right] \varphi[\tilde{v}] .
$$

Инвариантность меры дает $d[\tilde{v}]=d[v]$, откуда окончательно получаем

$$
\left(S_{1} \varphi\right)[u]_{\tau}=\int d[v] \exp \left[-i m \int_{0}^{\tau} d \sigma\left((u, v)+\frac{1}{2}(v, v)\right)\right] \varphi[v] .
$$

Оператор $S_{2} \in\left[U_{\chi}, U_{\kappa}\right]$, переплетающий локальное и элементарное представления в противоположном направлении, выводится совершенно аналогично:

$$
\left(S_{2} \psi\right)[v]=\int_{0}^{\infty} d \tau \int d[u]_{\tau} \exp \left[i m \int_{0}^{\tau} d \sigma\left((u, v)+\frac{1}{2}(v, v)\right)\right] \psi[u]_{\tau} .
$$

4.6. Пропагатор в пространстве траекторий. В соответствии с общей схемой теоретико-группового квантования (см. раздел 2) сконструируем теперь оператор $\Pi=S_{1} S_{2}$. Он переплетает локальное представление $U_{\chi}$ с ним самим, проецируя его на подпредставление, эквивалентное элементарному представлению $U_{\kappa}$. $\mathrm{C}$ помощью формул (23), (24) получаем

$$
\begin{aligned}
& (\Pi \psi)[u]_{\tau}=\int_{0}^{\tau} d \tau^{\prime} \int d[v] \exp \left[-i m \int_{0}^{\tau} d \sigma\left((u, v)+\frac{1}{2}(v, v)\right)\right] \psi[u]_{\tau^{\prime}}+ \\
& +\int_{0}^{\infty} d \tau^{\prime} \int d[v] \int d\left[u^{\prime}\right]_{\tau^{\prime}} \exp \left[i m \int_{0}^{\tau} d \sigma\left(\left(u^{\prime}, v\right)+\frac{1}{2}(v, v)\right)\right] \psi\left(\left[u^{\prime}\right]_{\tau^{\prime}}[u]_{\tau}\right) .
\end{aligned}
$$

Здесь $[u]_{\tau^{\prime}}, \tau^{\prime} \leqslant \tau$, обозначает начальную часть траектории $[u]_{\tau}$, т. е. часть, соответствующую интервалу $0 \leqslant \sigma \leqslant \tau^{\prime}$.

Таким образом, значение функции П $\psi$, соответствующее ее аргументу (т. е. траектории) $[u]_{\tau}$, выражается линейно через значения функции $\psi$, соответствующие различным траекториям $\left[u^{\prime \prime}\right]_{\tau^{\prime \prime}}$. Точнее, первый член формулы $(25)$ описывает вклад траекторий $\left[u^{\prime \prime}\right]_{\tau^{\prime \prime}}=[u]_{\tau^{\prime}}, \tau^{\prime \prime}=\tau^{\prime} \leqslant \tau$, а второй член - вклад траекторий $\left[u^{\prime \prime}\right]_{\tau^{\prime \prime}}=\left[u^{\prime}\right]_{\tau^{\prime}}[u]_{\tau}, \tau^{\prime \prime}=\tau+\tau^{\prime} \geqslant \tau$. Для нас сейчас важно, что в первом члене $\tau^{\prime \prime} \leqslant \tau$, тогда как во втором члене $\tau^{\prime \prime} \geqslant \tau$.

Параметр $\tau$ траектории $[u]_{\tau}$ в выражении $\psi[u]_{\tau}$ имеет смысл собственного времени, при котором описывается состояние $\psi$. Значит, первый член суммы описывает 
переход от состояния в более раннее собственное время к состоянию в более позднее значение собственного времени, т. е. причинный, каузальный переход. Наоборот, второй член - это переход от более позднего к более раннему собственному времени, т. е. акаузальный переход. Следовательно, наложение на оператор переплетения П принципа причинности (распространения лишь вперед по параметру собственного времени) должно состоять в отбрасывании второго, акаузального члена в формуле (25).

Это вполне соответствует введению множителя $\theta\left(x^{0}-x^{\prime 0}\right)$ в случае группы Галилея (см. вывод нерелятивистского пропагатора из группы Галилея в книге [5]). В релятивистском случае распространение вперед по собственному времени соответствует причинному пропагатору в пространстве-времени (см. п. 2.6).

На основе приведенных аргументов определим причинный (в смысле собственного времени) пропагатор в пространстве траекторий формулой

$$
\left(\Pi^{\mathrm{c}} \psi\right)[u]_{\tau}=\int_{0}^{\tau} d \tau^{\prime} \int d[v] \exp \left[-i m \int_{0}^{\tau} d \sigma\left((u, v)+\frac{1}{2}(v, v)\right)\right] \psi[u]_{\tau^{\prime}},
$$

где $[u]_{\tau^{\prime}}$ - начальная часть траектории $[u]_{\tau}$ (см. выше). Переход к обычному причинному пропагатору в пространстве-времени производится путем интегрирования по всем положительным значениями параметра собственного времени (см. п. 2.6).

В последнем выражении интегрирование по $d^{4} v(\sigma)$ при $-\infty<\sigma \leqslant \tau^{\prime}$ и при $\tau \leqslant \sigma<\infty$ дает числовой фактор, который можно опустить. Остающийся интеграл по скоростям $d^{4} v(\sigma), \tau^{\prime} \leqslant \sigma \leqslant \tau$, оказывается интегралом гауссова типа и может быть вычислен, что дает для причинного пропагатора в пространстве траекторий окончательное выражение

$$
\left(\Pi^{\mathrm{c}} \psi\right)[u]_{\tau^{\prime}}=\int_{0}^{\tau^{\prime}} d \sigma \exp \left(\frac{1}{2} i m \int_{\sigma}^{\tau^{\prime}} d \sigma^{\prime}(u, u)\right) \psi[u]_{\sigma}
$$

4.7. Пропагатор в пространстве-времени и калибровочные поля. Выше из теоретико-групповых соображений была введена функция $\psi[u]_{\tau}$. Она аналогична волновой функции, но только определена в пространстве траекторий, а не в пространстве-времени. Теперь мы перейдем к более привычному (и адекватному для точечных частиц) формализму, когда волновая функция зависит от точки пространства-времени и параметра собственного времени.

Величину $\psi[u]_{\tau}$ можно интерпретировать как амплитуду вероятности того, что частица движется вдоль данной траектории $[u]_{\tau}$, т. е. вдоль одной из кривых соответствующего класса, $\{x\}_{\tau^{\prime \prime}}^{\tau^{\prime}} \in[u]_{\tau}, \tau^{\prime}-\tau^{\prime \prime}=\tau$. Чтобы получить значение волновой функции в определенной точке $\left(x^{\prime}, \tau^{\prime}\right)$ пространства AРC, т. е. амплитуду вероятности нахождения частицы в этой точке, можно предположить, что частица выходит в момент собственного времени $\tau=0$ из некоторой фиксированной точки пространства-времени $x^{\prime \prime}$ (т. е. из точки $\left(x^{\prime \prime}, 0\right)$ пространства APC) и попадает в момент собственного времени $\tau^{\prime}$ в точку $x^{\prime}$ пространства-времени (т. е. в точку $\left(x^{\prime}, \tau^{\prime}\right)$ пространства АРС), двигаясь по одной из траекторий $[u]_{\tau^{\prime}}$. Учитывая, что амплитуда вероятности движения по траектории $[u]_{\tau^{\prime}}$ равна $\psi[u]_{\tau^{\prime}}$, и суммируя по всем траекториям, ведущим в точку $\left(x^{\prime}, \tau^{\prime}\right)$, получим в этом предположении для 
точечно-зависимой волновой функции выражение

$$
\Psi\left(x^{\prime}, \tau^{\prime}\right)=\int d[u]_{\tau^{\prime}} \psi[u]_{\tau^{\prime}}, \quad\left(x^{\prime}, \tau^{\prime}\right)[u]_{\tau}=\left(x^{\prime \prime}, 0\right)
$$

В этом рассуждении мы предполагали, что частица выходит из заданной точки пространства-времени, и находили амплитуду ее попадания в другие точки. Вместо этого можно сделать более общее предположение, фиксируя не отправную точку, а начальную волновую функцию. Пусть задано некоторое универсальное распределение $\Psi_{0}\left(x^{\prime \prime}, 0\right)$ по пространственно-временным точкам $x^{\prime \prime}$ в момент собственного времени $\tau=0$. Тогда точечно-зависимая волновая функция $\Psi\left(x^{\prime}, \tau^{\prime}\right)$ выражается через распределение по траекториям $\psi[u]_{\tau^{\prime}}$ (и универсальное первоначальное распределение $\left.\Psi_{0}\left(x^{\prime \prime}, 0\right)\right)$ формулой

$$
\Psi\left(x^{\prime}, \tau^{\prime}\right)=\int d\{x\}_{0}^{\tau^{\prime}} \alpha\{x\}_{0}^{\tau^{\prime}} \psi[u]_{\tau^{\prime}} \Psi_{0}\left(x^{\prime \prime}, 0\right)
$$

где через $\{x\}_{0}^{\tau^{\prime}}$ обозначен параметризованный путь, соединяющий точки $x(0)=x^{\prime \prime}$ и $x\left(\tau^{\prime}\right)=x^{\prime}$, а интегрирование ведется по всем таким путям (т. е. по соответствующим траекториям $\left.[u]_{\tau^{\prime}}, u(\sigma)=\dot{x}(\sigma)\right)$ с некоторым весовым функционалом $\alpha\{x\}_{0}^{\tau^{\prime}}$.

Для того чтобы формула (27) была корректна для всех значений собственного времени $\tau^{\prime}$, необходимо, чтобы функционал $\alpha\{x\}_{0}^{\tau^{\prime}}$ был частью представления $\alpha\{x\}_{\tau^{\prime \prime}}^{\tau^{\prime}}$ полугруппоида параметризованных путей, т. е. семейства операторов, удовлетворяющих условию мультипликативности

$$
\alpha\{x\}_{\tau^{\prime}}^{\tau} \cdot \alpha\{x\}_{\tau^{\prime \prime}}^{\tau^{\prime}}=\alpha\{x\}_{\tau^{\prime \prime}}^{\tau}
$$

с очевидными обозначениями.

Получающаяся функция $\Psi(x, \tau)$ соответствует тому же состоянию частицы, которое другим способом описывается распределением по траекториям $\psi[u]_{\tau}$, но функция $\Psi(x, \tau)$ описывает это состояние менее детально (одна функция $\Psi(x, \tau)$ соответствует целому классу функций $\left.\psi[u]_{\tau}\right)$.

Пусть $\Psi$ - состояние частицы, соответствующее распределению по траекториям $\psi$. Причинное распространение ведет тогда к другому состоянию, которое описывается распределением по траекториям $\Pi^{\mathrm{c}} \psi$ в соответствии с соотношением (26). С помощью формулы (27) можно найти соответствующее распределение по точкам пространства-времени $\Pi^{c} \Psi$ :

$$
\left(\Pi^{\mathrm{c}} \Psi\right)\left(x^{\prime}, \tau^{\prime}\right)=\int d\{x\}_{0}^{\tau^{\prime}} \alpha\{x\}_{0}^{\tau^{\prime}}\left(\Pi^{\mathrm{c}} \psi\right)[u]_{\tau^{\prime}} \Psi_{0}\left(x^{\prime \prime}, 0\right)
$$

Используя (26) и учитывая свойство мультипликативности представления $\alpha\{x\}_{\tau^{\prime \prime}}^{\tau^{\prime}}$ и меры $d\{x\}_{\tau^{\prime \prime}}^{\tau^{\prime}}$, можно привести это выражение к виду

$$
\left(\Pi^{c} \Psi\right)\left(x^{\prime}, \tau^{\prime}\right)=\int_{0}^{\tau^{\prime}} d \sigma \int d\{x\}_{\sigma}^{\tau^{\prime}} \exp \left[\frac{1}{2} i m \int_{\sigma}^{\tau^{\prime}} d \sigma^{\prime}(\dot{x}, \dot{x})\right] \alpha\{x\}_{\sigma}^{\tau^{\prime}} \Psi(\tilde{x}, \sigma),
$$


где обозначено $\tilde{x}=x(\sigma)$. Окончательно получаем формулу для причинного распространения пространственно-временной волновой функции в виде

$$
\begin{aligned}
\left(\Pi^{\mathrm{c}} \Psi\right)\left(x^{\prime}, \tau^{\prime}\right) & =\int d \tau^{\prime \prime} \int d x^{\prime \prime} \Pi^{\mathrm{c}}\left(x^{\prime}, \tau^{\prime} \mid x^{\prime \prime}, \tau^{\prime \prime}\right) \Psi\left(x^{\prime \prime}, \tau^{\prime \prime}\right) \\
\Pi^{\mathrm{c}}\left(x^{\prime}, \tau^{\prime} \mid x^{\prime \prime}, \tau^{\prime \prime}\right) & =\int d\{x\}_{\tau^{\prime \prime}}^{\tau^{\prime}} \exp \left[\frac{1}{2} i m \int_{\tau^{\prime \prime}}^{\tau^{\prime}} d \sigma(\dot{x}, \dot{x})\right] \alpha\{x\}_{\tau^{\prime \prime}}^{\tau^{\prime}}
\end{aligned}
$$

Здесь $\alpha\{x\}_{\tau^{\prime \prime}}^{\tau^{\prime}}$ должно быть представлением полугруппоида параметризованных пуmей. Нетрудно видеть, что такое представление может иметь вид ${ }^{13)}$

$$
\alpha\{x\}_{\tau^{\prime \prime}}^{\tau^{\prime}}=\exp \left\{i \int_{\tau^{\prime \prime}}^{\tau^{\prime}} d \sigma[V(x(\sigma), \sigma)+\mathbf{A}(x(\sigma), \sigma) \dot{x}]\right\}
$$

Такое определение при подстановке в формулу (29) приводит к пропагатору частицы во внешнем электромагнитном поле с электрическим потенциалом $V(x, \tau)$ и вектор-потенциалом $\mathbf{A}(x, \tau)$. Более общий вид представления полугруппоида параметризованных путей получается в том случае, когда $V(x, \tau)$ и $\mathbf{A}(x, \tau)$ - некоммутирующие операторы, а оператор $\alpha\{x\}_{\tau^{\prime \prime}}^{\tau^{\prime}}$ определяется как экспонента, упорядоченная вдоль кривой:

$$
\alpha\{x\}_{\tau^{\prime \prime}}^{\tau^{\prime}}=P \exp \left\{i \int_{\tau^{\prime \prime}}^{\tau^{\prime}} d \sigma[V(x(\sigma), \sigma)+\mathbf{A}(x(\sigma), \sigma) \dot{x}]\right\} .
$$

В этом случае формула (29) дает пропагатор во внешнем калибровочном поле.

Если предполагать, что пространственно-временное распределение выражается через распределение по траекториям формулой вида (27), то выражение (30) и вместе с ним формула (29) для пропагатора в пространстве-времени выводятся однозначно. Значит, использованный теоретико-групповой подход, дополненный этим предположением, приводит к выводу, что никаких полей, кроме калибровочных, быть не может. На самом деле вместо (27) можно принять более общее выражение для пространственно-временной волновой функции, и тогда в рамках данного подхода можно получить не только калибровочное, но и гравитационное поле, т. е. описать движение частицы в искривленном пространстве-времени. Формула, заменяющая (27), будет в этом случае включать неголономное описание сдвигов в пространстве-времени, использующее операцию параллельного переноса в расслоении реперов над искривленным пространством-временем [10]. Мы не будем здесь более детально рассматривать эту возможность.

Заметим в заключение, что к волновой функции и пропагатору, найденным в настоящем разделе, применимо все, что сказано в п. 3.4 о выделении определенного спина из всех спинов, описываемых неприводимым представлением $\Delta$ группы Лоренца. В соответствующих дифференциальных операторах вместо обычных производных следует использовать ковариантные производные, возникающие в калибровочном и/или гравитационном поле. При этом возникает произвол, связанный с некоммутативностью ковариантных производных.

13) Ниже мы упомянем "неголономное” обобщение этого представления, описывающее гравитационное поле. 


\section{5. ЗАКЛЮЧИТЕЛЬНЫЕ ЗАМЕЧАНИЯ}

В настоящей работе показано, что процедуру теоретико-группового вывода динамики можно применить не только к нерелятивистским (как в работе [6]), но и к релятивистским объектам. Исходным пунктом для этого являются группа АРС (релятивистское обобщение группы Галилея) и обобщенная полугруппа АРС (получающаяся заменой пространственно-временных сдвигов на траектории в пространстве-времени).

Динамика частицы возникает при этом в форме фейнмановского интеграла по путям, который является следствием трех теоретико-групповых фактов:

1) инвариантная мера на пространстве траекторий имеет вид (20);

2) обобщенная полугруппа АРС имеет проективные представления, мультипликаторы которых в конечном счете приводят к весовому функционалу вида $e^{i S}$ в интеграле по путям (26), где $S$ - функционал действия свободной частицы;

3) выражение (27) для точечно-зависимой волновой функции содержит представление $\alpha\{x\}_{\tau^{\prime \prime}}^{\tau^{\prime}}$ полугруппы траекторий, а такое представление, как оказывается, описывает внешнее калибровочное поле (30).

Тот факт, что состояние частицы в этом формализме может быть охарактеризовано не только пространственно-временным распределением $\Psi$, но также распределением по траекториям $\psi$, является очень важным. Поскольку группа путей и ее модификации естественным образом описывают фундаментальные понятия в квантовой гравитации (см. раздел 1), появление путезависимой волновой функции $\psi$ можно интерпретировать не просто как промежуточный объект, но как указание на истинный нелокальный характер описываемых квантовых объектов, которые лишь в специальных условиях ведут себя как точечные частицы.

Формула (26) показывает, что с течением собственного времени $\tau$ траектория $[u]_{\tau}$, описывающая конфигурацию нелокального объекта, наращивается. Такие объекты можно интерпретировать как "струны-истории", вытянутые не в трехмерном пространстве, а в пространстве-времени. В книге [10] (более детально - в ее расширенном переводе на японский язык) было показано, что модель, в которой кварки представляются калибровочно заряженными "струнами-историями", объясняет феномен их удержания (конфайнмента). Глюонное поле в этом случае также описывается нелокальными объектами ${ }^{14)}$ (см. статью [19]).

Указание на фундаментальный характер нелокальных черт теории можно видеть и в том, что динамика, выраженная в терминах путезависимой волновой функции $\psi$, оказывается тривиальной (описывается лишь кинетической частью действия, см. формулу (26)). Взаимодействие с внешними полями появляется (в формуле (30)) лишь в том случае, когда мы для описания состояний пользуемся функцией $\Psi$, зависящей от пространственно-временных точек. Это очевидным образом перекликается с тем фактом, что формализм путезависимых волновых функций является явно калибровочно-инвариантным, тогда как при использовании функций, зависящих от точек, т. е. в явно локальной теории, остается произвол выбора калибровки, и необходимо налагать условие калибровочной инвариантности наблюдаемых величин ${ }^{15)}$.

14) Нелокальные объекты, моделирующие глюоны, представляются группой 2-путей вместо обычной группы путей. 
Отметим, что кроме калибровочного поля, появляющегося в формуле (30), можно ввести и гравитационное поле, т. е. описать движение нашего объекта в искривленном пространстве-времени. Представление полугруппы траекторий, входящее в определение пространственно-временной волновой функции, должно тогда строиться с помощью операции параллельного переноса в расслоении реперов над искривленным пространством-временем [10]. Мы не делаем этого здесь в явном виде, потому что полугруппа траекторий действует на искривленном пространствевремени неголономно (т. е. обычным образом действует не в самом пространствевремени, а в расслоении над ним). Технически это сложнее, чем описание калибровочного поля.

Благодарности. Автор признателен И. В. Тютину, который прочел статью в рукописи и сделал ряд замечаний, способствовавших устранению некоторых неточностей и улучшению формулировок.

\section{Список литературы}

[1] E. Wigner, Ann. Math., 40:1 (1939), 149-204.

[2] T. Newton, E. Wigner, Rev. Modern Phys., 21:3 (1949), 400-406.

[3] A.S. Wightman, Rev. Modern Phys., 34:4 (1962), 845-872.

[4] M. B. Mensky, Commun. Math. Phys., 47:2 (1976), 97-108.

[5] М.Б. Менский, Метод индуцированных представлений: пространство-время и концепция частии, Наука, М., 1976.

[6] М. Б. Менский, ТМФ, 57:2 (1983), 217-231.

[7] S. Mandelstam, Ann. Phys., 19:1 (1962), 1-24; 25-66.

[8] S. Mandelstam, Phys. Rev., 175:5 (1968), 1580-1603; 1604-1623.

[9] М. Б. Менский, ТМФ, 18:2 (1974), 190-202.

[10] М. Б. Менский, Группа путей: измерения, поля, частичъ, Наука, М., 1983.

[11] M. Süveges, Acta Phys. Acad. Sci. Hung., 20 (1966), 41-50; 20 (1966), 51-58; 22 (1966), 273-284; 27 (1969), 261-268.

[12] M. B. Mensky, Lett. Math. Phys., 2:3 (1978), 175-180.

[13] M. B. Mensky, Lett. Math. Phys., 3:6 (1979), 513-520.

[14] M. B. Mensky, Helv. Phys. Acta, 69:3 (1996), 301-304, arXiv: gr-qc/0010105.

[15] J. J. Aghassi, P. Roman, R. M. Santilli, Phys. Rev. D, 1:10 (1970), 2753-2765.

[16] E. C. Stueckelberg, Helv. Phys. Acta, 14 (1941), 321-322; 15 (1942), 23-37.

[17] J. J. Aghassi, P. Roman, R. M. Santilli, J. Math. Phys., 11 (1970), 2297-2301.

[18] J. J. Aghassi, P. Roman, R. M. Santilli, Nuovo Cimento A, 5:4 (1971), 551-590.

[19] М. Б. Менский, ЖЭТФ, 90:2 (1986), 416-428.

Поступила в редакцию 24.05.2012, после доработки 18.07.2012

15) С математической точки зрения такого рода факты можно рассматривать как пример тривиализации динамики при введении вспомогательных степеней свободы. Другие примеры этого типа известны из теории вполне интегрируемых систем. 